\title{
Densification Behaviour in the Fabrication of Al-Fe Metal Matrix Composite Using Powder Metallurgy Route
}

\author{
Siddhartha Tiwari, ${ }^{1,2}$ Priyanka Rajput, ${ }^{1}$ and Sanjay Srivastava ${ }^{1}$ \\ ${ }^{1}$ Department of Materials Science and Metallurgical Engineering, University Institute of Engineering \& Technology, \\ CSJM University, Kanpur 208024, India \\ ${ }^{2}$ Department of Metallurgical and Materials Engineering, Indian Institute of Technology Kharagpur, Kharagpur 721302, India
}

Correspondence should be addressed to Siddhartha Tiwari, iitkgp.siddhartha@gmail.com

Received 19 September 2012; Accepted 8 October 2012

Academic Editors: Y. S. Chang and S. C. Wang

Copyright (c) 2012 Siddhartha Tiwari et al. This is an open access article distributed under the Creative Commons Attribution License, which permits unrestricted use, distribution, and reproduction in any medium, provided the original work is properly cited.

Densification behaviour of the powder particles in two- and/or multicomponent system is unpredictable. The present work deals with the densification behaviour of Al-Fe powder particles during compaction and sintering in order to fabricate the Al-Fe metal matrix composites by powder metallurgy route. Green compacts of Al-6.23 wt.\% Fe powder particles were fabricated under varying compaction pressures, and these fabricated green compacts were sintered over a series of temperatures $\left(430^{\circ} \mathrm{C}-590^{\circ} \mathrm{C}\right)$. The sintered products have been characterised with the help of X-ray diffraction (XRD) and scanning electron microscope attached with energy dispersive spectroscopy (EDS). It has been found that the green density of the compacts increases with increase in compaction pressure. The rate of change in green density is found to be lower at higher compaction pressures. Sintered density increases with increasing sintering temperature up to $550^{\circ} \mathrm{C}$ whereas a drop in sintered density is perceived at $590^{\circ} \mathrm{C}$. This decrease in sintered density is considered to occur due to swelling which has been explained on the basis of the Kirkendall effect. The XRD and EDS analyses of sintered products indicated the presence of $\mathrm{Al}$ and Fe particles with the trace amount of intermetallics.

\section{Introduction}

Aluminium matrix composites (AMCs) are one of the most demanding engineering materials in the category of metal matrix composites (MMCs) due to combination of their light weight and excellent mechanical and tribological properties. These composites have been widely used for structural, nonstructural, and functional applications in automotive and aerospace industries [1-3]. Powder metallurgy (P/M) route is one of the most widely used methods for producing AMCs due to its low processing costs as well as the ease and the accuracy to obtain near net shaped components of complex geometry. The most essential steps of $\mathrm{P} / \mathrm{M}$ technique are mixing of powders, compaction, and sintering [4-6]. Compaction of loose powders under externally applied pressure and sintering of green compacts produced in earlier stage are the main processing parameters, and these parameters affect the density of the final product. Densification behaviour under compaction depends mainly on the applied pressure and yield stress of the powder particles. One of the important processing steps in $\mathrm{P} / \mathrm{M}$ route is sintering, which is used to produce density-controlled materials and components from metal and/or ceramic powders by applying thermal energy. It can be divided into two categories: solid-state sintering and liquid-state sintering, below and above the melting temperature respectively [7].

It is easy to predict the densification behaviour of singlecomponent powder particles where the hardness and/or yield stress of powder particles is approximately the same. But, in two or multicomponent systems, where the hardness of each of the powder particles is different, it is difficult to apply any generalised theory or model [8]. Bouvard [8] briefly described the densification behaviour of hard and soft powders under applied pressure in order to fabricate metal ceramic composites and concluded that the mechanism of densification varies with the amount of load applied relative to the hardness of the powder particles. A number of 
models have been proposed to understand compaction of loose powder and densification during sintering, but most of the models are based on single-component system [912]. Interestingly, proposed models and theories of sintering construct fundamental ideas to understand sintering behaviour of one, two, and/or multiphase system, but in practice sintering behaviour of two or multicomponent system is unpredictable, as it may be accompanied with shrinkage, swelling, or no volume change [13-15]. For example, Kown and savitskii [15] summarised the experimental results related to the densification behaviour of two-component systems during solid-state sintering such as $\mathrm{W}-\mathrm{Fe}, \mathrm{Cr}-\mathrm{Ni}$, Cr-Pd, Co-Mo, $\mathrm{Cu}-\mathrm{Ag}$, Ni-Mo, Co-Fe, Fe$\mathrm{Co}$, and $\mathrm{Fe}-\mathrm{Cu}$ and discussed the sintering mechanisms. It is noticed that in all the above binary component systems, the densification characteristics during sintering, like swelling, compaction, and/or no volume change, are strongly dependent on the concentration of the elements and exhibit different densification behaviour in different systems. Therefore in $\mathrm{P} / \mathrm{M}$ process, to achieve optimum density of a particulate composite of two-component system, it is necessary to construct preliminarily investigations on the densification behaviour with the help of experimental results. In a comprehensive study, it is found that most of the earlier investigations, related to Al-Fe binary phase system, are focused either on liquid-phase sintering and/or iron-rich composition (more than $20 \mathrm{wt} . \% \mathrm{Fe}$ ) $[2,16]$. Therefore in the present investigation, an attempt has been made to examine the compaction and sintering behaviour of Al-rich $\mathrm{Al}-\mathrm{Fe}$ binary phase system.

This experimental study deals with the densification behaviour of mixture of $\mathrm{Al}-6.23 \mathrm{wt} . \% \mathrm{Fe}$ powders under compaction and sintering to produce Al-Fe metal matrix composite by $\mathrm{P} / \mathrm{M}$ route. The major objectives of present investigation are to study (a) the effect of compaction pressure on the green density of compacts, prepared under 133$399 \mathrm{MPa}$ compaction pressure and (b) the effect of sintering temperature on the densification of each green compacts in the temperature range of $430^{\circ} \mathrm{C}-590^{\circ} \mathrm{C}$. The microstructures of sintered products have been characterised using scanning electron microscope (SEM) and energy dispersive spectroscopy (EDS). X-ray diffraction (XRD) analysis has been carried out to examine the existing phases after sintering. The obtained results are briefly explained to understand the mechanisms involved during the densification under compaction and sintering. The swelling behaviour at higher sintering temperature is also discussed.

\section{Experimental}

Commercially atomized aluminium powder with $99.7 \%$ purity and electrolytic iron powder with $99.5 \%$ purity of 250-300 mesh size (supplied by Loba Chemie Private Ltd., India) have been selected for the present investigation. The appropriate amount (Al-6.23 wt.\% Fe) of selected powders was properly mixed for approximately 150-180 minutes, using agate mortar and pestle. The mixture of powders (approximately $1 \mathrm{gm}$ ) was then subjected to compaction at pressure range of 133-399 MPa to prepare the cylindrical pellets (green compacts) of $13 \mathrm{~mm}$ diameter and $3 \mathrm{~mm}$ height. A single acting hydraulic press was used for compaction, and zinc stearate was sprayed on the inner walls of die for lubrication. Weighing was done using electronic pan balance. The prepared green compacts were then dried for 30 minutes at temperature $200^{\circ} \mathrm{C}$ for complete removal of moisture, entrapped gases, and other impurities. Furthermore, sintering of all the green compacts was carried out using a ceramic tubular furnace in argon atmosphere at a heating rate of $5^{\circ} \mathrm{C} / \mathrm{min}$. To investigate the variation in sintered density and change in phases, these prepared pellets were sintered for 120 minutes at temperatures $430^{\circ} \mathrm{C}, 470^{\circ} \mathrm{C}, 510^{\circ} \mathrm{C}, 550^{\circ} \mathrm{C}$, and $590^{\circ} \mathrm{C}$ followed by furnace cooling. Theoretical density and porosity were calculated using the following equations:

Theoretical density

$$
=\frac{(\text { Density of } \mathrm{Fe} \times \% \mathrm{Fe})+(\text { Density of } \mathrm{Al} \times \% \mathrm{Al})}{100},
$$

Porosity

$$
=\left(\frac{\text { Theoretical density }- \text { Experimental density }}{\text { Theoretical density }}\right) \times 100
$$

The green and sintered densities of pellets were measured by weight/volume ratio as well as the Archimedes principle (water displacement technique) to ensure the appropriateness of values of densities. The microstructure of the prepared composite was examined with the help of a scanning electron microscope (SEM, model: JEOL, JSM5800 , JEOL Ltd., Japan). The elemental analysis of the specimens was performed using an energy dispersive Xray (EDX) microanalyser equipped with scanning electron microscope (SEM). Metallographic samples were prepared by standard procedure, that is, first ground up to 400 grade emery papers and then polished up to $0.25 \mu \mathrm{m}$ diamond finish using Meta Serve 250 Grinder-Polisher made by BUEHLER group. The polished samples were then etched by Keller's reagent. X-ray diffraction (XRD) analyses have been performed for the identification of the phases with PHILIPS PW1792 diffractometer, operated with $\mathrm{Cu} \mathrm{K \alpha}$ radiation. The obtained peaks, corresponding to the phases, were identified by matching with the JCPDS standards using X'Pert High Score Plus software.

\section{Results and Discussion}

3.1. Effect of Compaction Pressure: Variation in Green Density. The study deals with the densification behaviour of Al-rich Al-Fe metal matrix composite during sintering. Al-Fe green compacts of well-mixed $\mathrm{Al}$ and $\mathrm{Fe}$ powders (6.23 wt.\% Fe) were prepared. Theoretical density of these green compacts was calculated to be $3.02 \mathrm{~g} / \mathrm{cm}^{3}$. The compaction pressure within the range of 133-399 MPa was applied for preparing the green compacts. Figure 1(a) shows the variation in green density and Figure 1(b) illustrates the relative change in theoretical density (compaction) as well as relative change in 


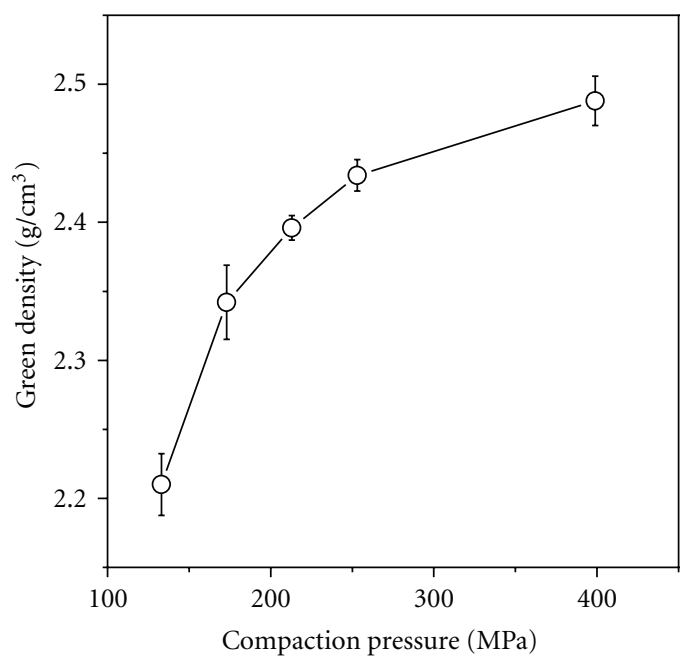

(a)

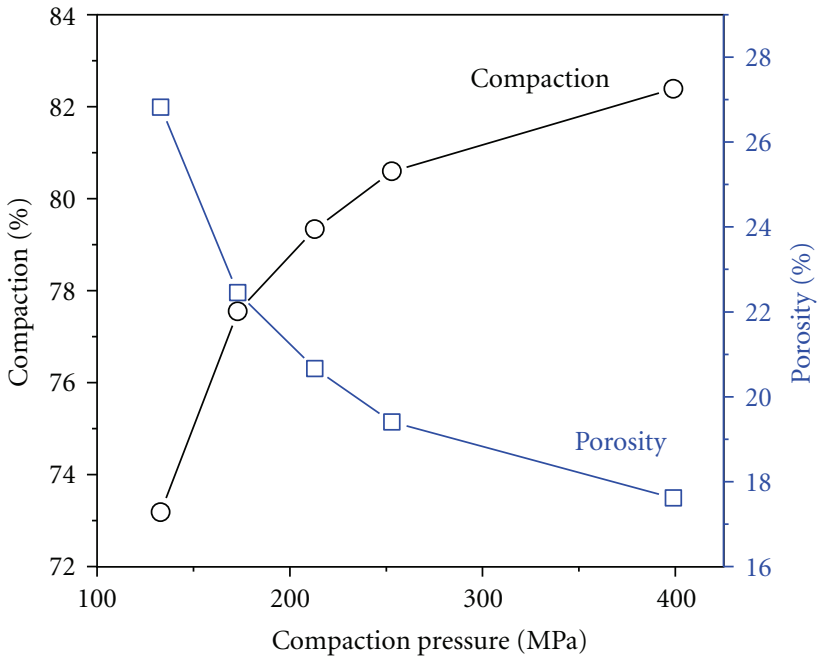

(b)

FIGURE 1: Variation in (a) green density and (b) compaction (theoretical density) with respect to compaction pressure.

porosity with respect to compaction pressure. The maximum green density of the prepared green compact is found $\sim 2.5 \mathrm{~g} / \mathrm{cm}^{3}$ at $399 \mathrm{MPa}$ pressure which is $\sim 82 \%$ of the theoretical density. The percentage change in theoretical density of the green compacts is termed as compaction. It is found that the green density of the compacts increases with increasing compaction pressure, but the rate of increase in green density decreases with the increase in compaction pressure. A similar kind of trend is reported earlier also [8, 17]. The most basic and efficient processes that occur during compaction under pressure are the bulk movement or the rearrangement of particles and the deformation of particles. Bulk movement of particles depends upon the particle to particle contact and free space into which the particle may move. This movement is limited by the frictional forces developed between neighbouring particles and between particles and die surfaces. Therefore, the relative ease of such motion increases with decrease in apparent density and particle size. In the case of die compaction, there is some lateral motion due to restraining action of blocking particles and the availability of free spaces. The bulk movement of the particles is thus restrained by the powder characteristics which increase the frictional forces. The movement of particles within the powder mass takes place at relatively low pressures and accounts for the early densification of the material. The densification during compaction due to plastic deformation of individual particles is also an important mechanism. In this densification process the effectiveness of external pressure is highest at low pressures and becomes progressively less effective with increasing pressure $[8,17$, 18].

A relative decrease in porosity from $\sim 27 \%$ to $\sim 17 \%$ can also be seen in Figure 1(b) with increase in compaction pressures and green density. Generally, in case of pure aluminium, around $90 \%$ of theoretical density can be achieved in the selected pressure range, but due to the presence of the iron particles in the Al-Fe powder mixture, only $\sim 82 \%$ of theoretical density is achieved. As aluminium particles are soft and have lower yield strength with regards to the applied pressures, the soft aluminium particles play the major role in the densification. On the other hand the iron particles are relatively hard and hinder the densification $[8,17,19]$.

\subsection{Effect of Sintering Temperature: Variation in Sintered} Density. The densification behaviour during sintering of selected $\mathrm{Al}-\mathrm{Fe}$ green compacts is illustrated in Figure 2. Figure 2(a) represents variation in sintered density with respect to green density at each sintering temperature. It is observed that the rate of change of sintered density is higher for the compacts of lower green density whereas its magnitude is lower for the compacts of higher green density. The reason behind this kind of behaviour is associated with the driving force required for sintering. In the earlier stages of sintering, the driving force is produced by the surface energy, which is associated with the internal surface area of the particles. For sintering, a decreasing green density signifies an increase in the amount of internal surface area and, consequently, a greater driving force. Therefore, rate of change in sintered density resulting from Figure 2(a) increases with decrease in green density [7].

In Figure 2(b), it has been found that the sintered density increases with increase in sintering temperature up to $550^{\circ} \mathrm{C}$ (which is $75 \%$ of the melting temperature of the matrix); however, a decrease in sintered density is observed above this temperature for all the green compacts. Since sintered density is associated with the dimensional changes of green compacts, therefore it can be stated that the volume shrinkage is taking place up to $550^{\circ} \mathrm{C}$ whereas a swelling of compact is taking place at $590^{\circ} \mathrm{C}$. It is difficult to generalise any theory to describe this kind of sintering behaviour of two- or multicomponent system. In general, sintering involves initial bonding among particles, neck growth, pore channel closure, 


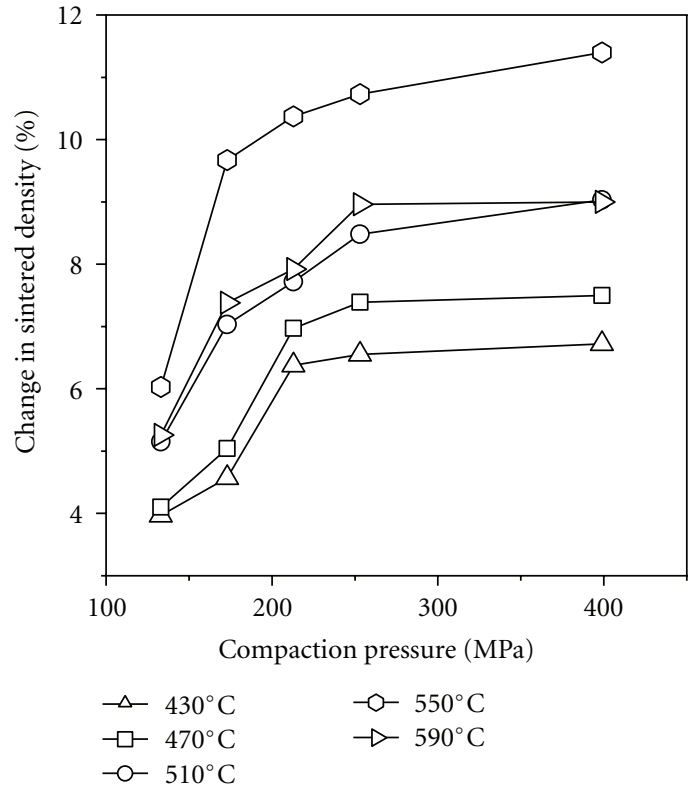

(a)

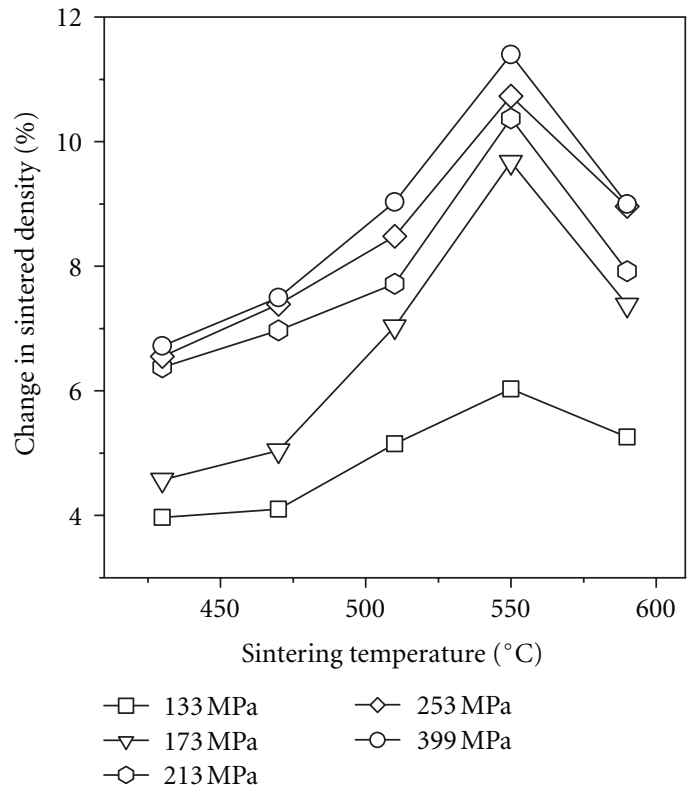

(b)

FIGURE 2: Variation in \% change in sintered density with respect to (a) compaction pressure and (b) sintering temperature.

pore rounding, and densification or pore shrinkage. The alliance of these stages is basically accomplished by lowering of free energy of the system which is associated with the diffusion of particles and vacancy $[8,13-15]$. Various models have been proposed for the neck formation and growth of spherical particles due to vacancy and grain boundary diffusion $[20,21]$.

In actual practice, particles associated with the sintering mechanism are irregular in size and morphology. The basic mechanism which involves transportation of the matter during sintering is proposed by diffusion process for both singleand two-component systems and can be well described with the help of the Kirkendall effect $[15,22]$. Here, up to a certain temperature range, volume shrinkage takes place, and after that temperature, volume expansion is observed which can also be pronounced as swelling. The Kirkendall effect is nothing but diffusion of vacancies and/or atoms due to concentration and/or temperature gradient [23]. In the present situation, this expected mechanism is taking place between aluminium-aluminium particles and aluminiumiron particles. In both cases, grain boundaries and exterior surfaces are probable positions for both the creation and elimination of vacancies $[15,24]$. The experimental facts best concur the concept that is the most important mechanism for the creation of vacancies is dislocation climb and that dislocation climb and void formation account for most of the absorbed vacancies, positive climb being associated with the removal of vacancies and negative climb with the creation of vacancies. In aluminium, vacancy diffusion is the most favourable process, because in face centred cubic (FCC) system, the least amount of thermal energy is required to activate the vacancy movement [25]. Let us assume a situation when two aluminium particles are in contact with each other (no grain boundary between the particles) and a small neck of high curvature forms. This high curvature is the main cause for the vacancy accumulation near the neck region and results into a concentration gradient of vacancies, which provides a driving force for diffusion. Vacancies moving away from the neck translate material moving towards the neck, and neck radius rapidly increases as material is deposited there, which results in neck growth and system shrinkage [20]. If the particles are dissimilar in size, then differences in curvature along the particle surface produce stresses that produce a difference in chemical potential. These chemical potential differences are translated to vacancy concentration differences. In most of the cases where single-component system is considered, volume shrinkage takes place. It is also well known that sintering is extremely dependent on temperature. An increase in the sintering temperature greatly increases the rate and magnitude of any changes occurring. At higher temperature, translation of vacancies increases which helps in increase of density and/or volume shrinkage $[15,20,21]$.

In body-centered cubic (BCC) metals like $\alpha$-iron, diffusion by ring mechanism requires less thermal energy than vacancy diffusion; the discovery of the Kirkendall effect in BCC metals indicates that these metals also diffuse by a vacancy mechanism [23]. Now the question arises, how a diffusion mechanism takes place between two particles of different materials where substitutional solid solution has been produced as a result of formation of intermetallic compound, which also may be a cause of swelling $[16,21]$. This type of mechanism is well described by Kwon and savitskii [15] with the help of the Kirkendall effect for many binary metal powder mixtures. In this proposed mechanism, concentration stresses arise between two different powder 


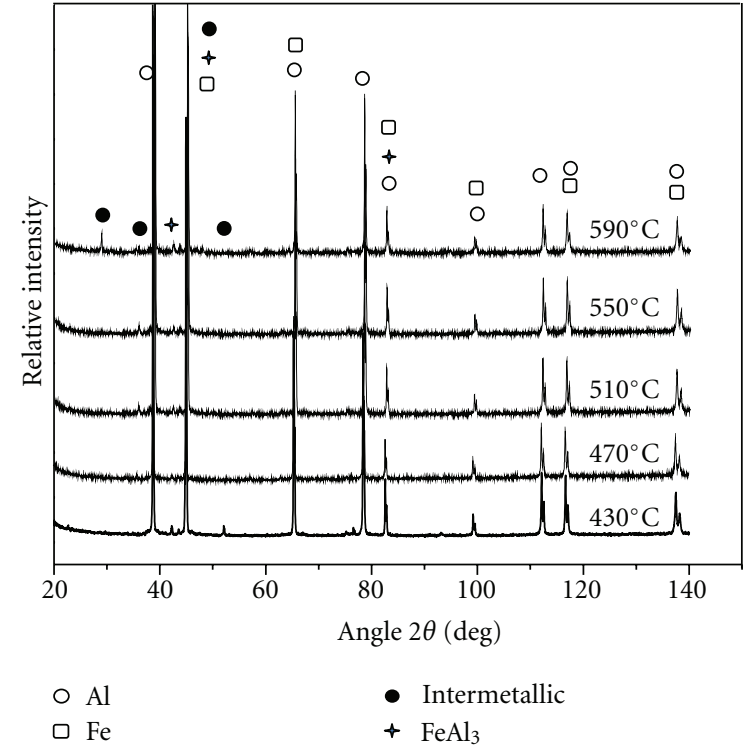

Figure 3: The XRD results of Al-6.23wt.\% Fe compacted at $399 \mathrm{MPa}$ and sintered at various temperatures for 120 minutes.

components mixed in diffusion zone because of the differences in type of crystal lattice, lattice parameters, elastic modules, and so on. Also, the presence of grain boundaries between the particles acts as a sink for vacancies $[15,21]$. Diffusion process is motivated by driving forces which come up due to temperature gradient, concentration gradient, mobility of atoms, and so forth. The Kirkendall experiment demonstrates the rate at which the diffusion of two types of atoms of binary solution is not the same; therefore mobility of atoms is one of the important parameters [23]. In Al-Fe powder mixture, aluminium has lower melting point than iron; therefore when temperature is raised up to $590^{\circ} \mathrm{C}$, the mobility of aluminium particles increases drastically as this temperature is close to the melting temperature of aluminium. High diffusivity of aluminium [26] causes more vacancy creation which results in void formation as well as substitutional solid solution of Al-Fe particles.

3.3. X-Ray Diffraction and Microstructural Analyses. XRD analysis has been carried out in order to find out the phases present in a sintered product. It should be noted that as illustrated in Section 3.2 (Figure 2(b)), green compacts prepared under the compaction pressure of $399 \mathrm{MPa}$ produced maximum change in sintered density in the temperature range of $430^{\circ} \mathrm{C}$ to $590^{\circ} \mathrm{C}$. Therefore, only the above specimens have been considered for further studies like phase, elemental, and microstructural analyses. The diffraction patterns of the selected samples are shown in Figure 3.

The most prominent and relatively high intense peaks of $\mathrm{Al}$ and $\alpha$-Fe were identified, as matched with the JCPDS file no. 00-001-1180 for Al and 00-006-0696 for Fe. Relatively high intensities of $\mathrm{Al}$ and $\alpha$-Fe corresponding to their utmost amount in sintered products. On the other hand, a number of tiny peaks correspond to the intermediate phases like $\mathrm{FeAl}_{3}$ (JCPDS file no. 00-001-1265) have been examined. Interestingly, the existence of some new peaks has been found at $590^{\circ} \mathrm{C}$ which does not have any correspondence to either $\mathrm{Al}$ or $\mathrm{Fe}$ and neither with aluminium oxide. On rigorous analysis, the closest match found to the referred peaks was of $\mathrm{Al}_{13} \mathrm{Fe}_{4}$ (JCPDS file no. 00-050-0797), but any strong evidence of existence of $\mathrm{Al}_{13} \mathrm{Fe}_{4}$ phase is still unavailable. Although these peaks were absent at $430^{\circ} \mathrm{C}$ and $470^{\circ} \mathrm{C}$, a deficient amount has been noticed at $510^{\circ} \mathrm{C}$ and $550^{\circ} \mathrm{C}$. Consequently, appearance of this intermediate phase at $590^{\circ} \mathrm{C}$ could be a decisive cause of decrease in sintered density (swelling).

A brief study of earlier investigations related to $\mathrm{Al}-\mathrm{Fe}$ binary system describes detailed ideas regarding the formation of intermetallic compounds, produced by a variety of techniques like mechanical alloying and solid- and liquidphase sintering. For example, Kang and $\mathrm{Hu}$ [16] reported the swelling behaviour of reactive sintering of Fe-29 at.\% $\mathrm{Al}$ mixtures due to the formation of an intermediate phase having chemical formula $\mathrm{Fe}_{2} \mathrm{Al}_{5}$ at the temperature range of $500^{\circ} \mathrm{C}-750^{\circ} \mathrm{C}$. One of the authors of the present investigation has already reported the formation of $\mathrm{Al}_{3} \mathrm{Fe}$ intermetallics in the Al-Fe composites which has been prepared by dispersion of iron in aluminium melt by impeller mixing and bottom pouring chill casting technique [2]. The iron content for the above investigation varies from 1.67 to $11.2 \mathrm{wt} \%$. This report suggests that the formation of intermetallics can also take place at low concentration of iron in aluminium-iron binary mixtures. Olszówka-Myalska et al. [27] characterised intermetallic compounds in a aluminium-iron metal matrix composite in the composition range of 3-6 vol.\% Fe at hot pressing temperature of $630^{\circ} \mathrm{C}$ for the time duration of 5-30 minutes. But they have not mentioned the chemical formula of the characterised intermetallic compound. In a study of structural evaluation of mechanically alloyed $\mathrm{Al}-\mathrm{Fe}$ powders, the authors reported a mixed amorphous phase and solid solution of $\mathrm{Fe}$ in $\mathrm{Al}$, having a composition of $\mathrm{Al}-10$ at.\% Fe. It has also been recommended that the formation of $\mathrm{FeAl}_{3}$ intermetallic can take place after heat treatment of milled powders within a range of 4 to 10 at.\% $\mathrm{Fe}$ [28]. As discussed in Section 3.2, in solid-state sintering, formation of intermetallic compound takes place due to interdiffusion of atoms. This interdiffusion strongly depends on diffusivity of each element. Although iron has extremely low solubility in aluminium, the diffusivity of iron in aluminium was found to be maximum at temperature range $550^{\circ} \mathrm{C}-630^{\circ} \mathrm{C}$ in an investigation carried out from $350^{\circ} \mathrm{C}$ to $630^{\circ} \mathrm{C}$ [26].

Moreover, in the diffusion couple of aluminium and iron, aluminium can move faster than iron because of its low melting temperature. On the basis of earlier reports and the present investigation, it can be emphasized that the formation of intermetallic compounds or solid solution can take place in aluminium-rich composition of aluminiumiron composites or alloys below the melting point of aluminium. Here, it is expected that the formation of a new phase is taking place at $590^{\circ} \mathrm{C}$ in a considerable amount and a remarkable intense peak of intermetallic most likely $\mathrm{Al}_{13} \mathrm{Fe}_{4}$ has been marked. Hence, the existence of this kind of solid 


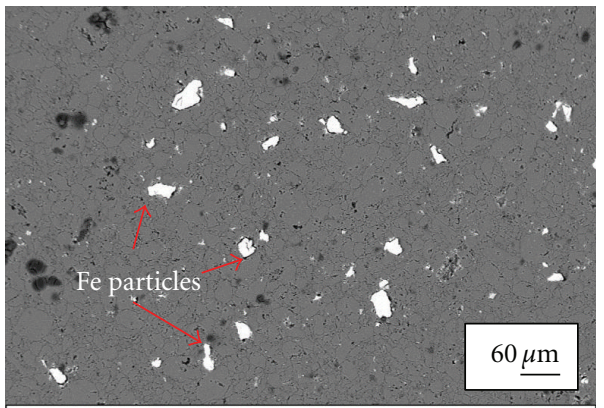

(a)

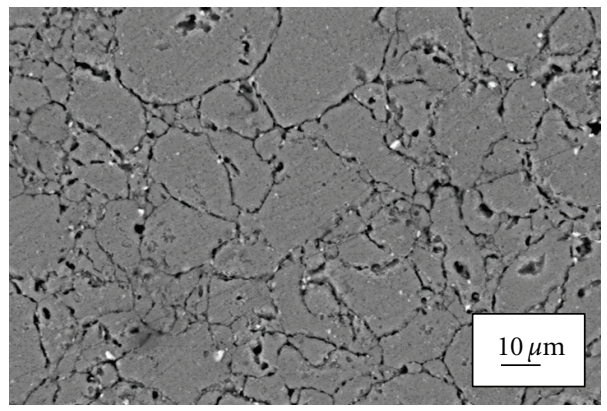

(c)

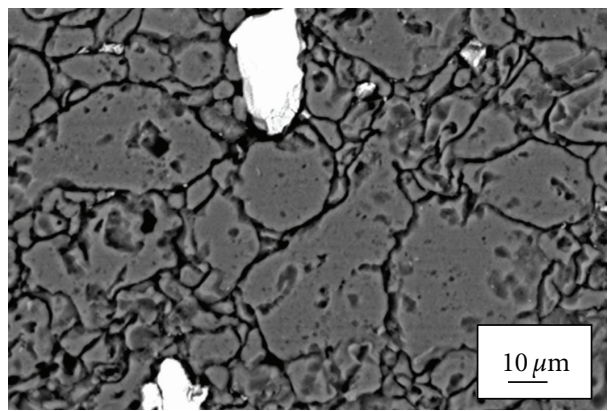

(b)

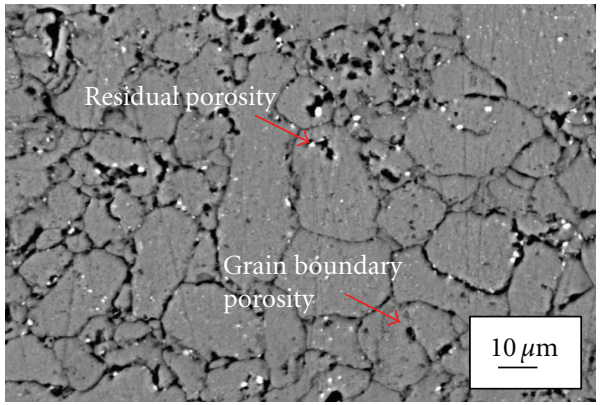

(d)

FIGURE 4: Typical SEM microstructure of Al-Fe composite shows (a) distribution of Fe particles over $\mathrm{Al}$ matrix sintered at $550^{\circ} \mathrm{C}$, residual and grain boundary porosities (b) sintered at $510^{\circ} \mathrm{C}$, (c) sintered at $550^{\circ} \mathrm{C}$, and (d) $590^{\circ} \mathrm{C}$.

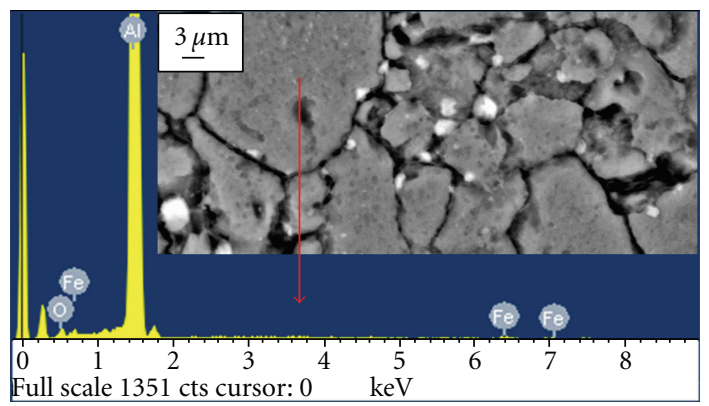

(a)

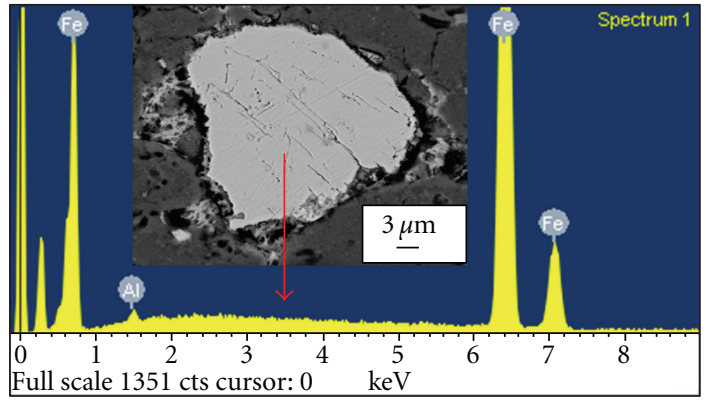

(b)

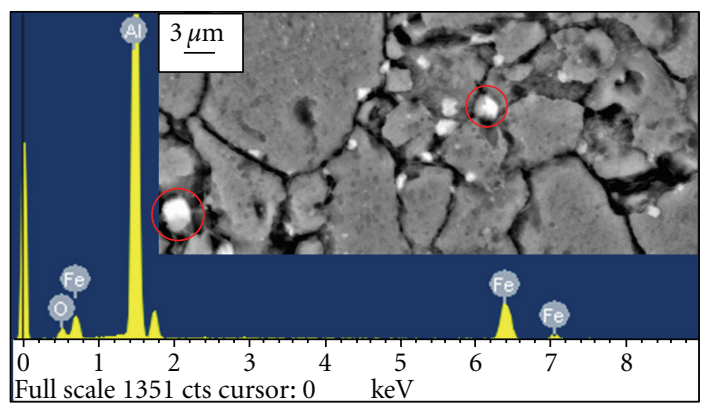

(c)

FIGURE 5: EDS spot analysis of sintered Al-Fe metal matrix composite: (a) Al matrix, (b) Fe particle consists small amount of Al, and (c) small particles (intermetallics) exhibit considerable amount of $\mathrm{Al}$ and $\mathrm{Fe}$. 
solution can also account as a reason for decreased sintered density at this temperature.

Figure 4 represents the SEM images of the Al-Fe sintered product. The distribution of $\mathrm{Fe}$ in $\mathrm{Al}$ matrix sintered at $550^{\circ} \mathrm{C}$ can be easily seen in Figure $4(\mathrm{a})$ at relatively lower magnification. Figures 4(b)-4(c) show the matrix of sintered product, sintered at $510^{\circ} \mathrm{C}, 550^{\circ} \mathrm{C}$, and $590^{\circ} \mathrm{C}$, respectively. It is also noticed that very tiny iron particles are distributed throughout the aluminium matrix along the grain boundary and throughout the grains. Microstructures also represent reduction of porosity along the grain boundaries and residual porosity within grains. The concentration of the residual and grain boundary porosity is noticed more in the microstructure of specimen sintered at $590^{\circ} \mathrm{C}$.

The EDS profile corresponding to the microstructures is also given as an evidence of elements present in the sintered product. Figure 5 illustrates the points at which EDS is performed, and the intensity of peaks is corresponding to the elements present at that point. The existence of elements in all possible phases such as presence of $\mathrm{Al}$ in $\mathrm{Fe}$ particle and $\mathrm{Fe}$ in Al matrix is shown in Figures 5(a) and 5(b), respectively. The EDS result of tiny particles shows existence of both $\mathrm{Al}$ and $\mathrm{Fe}$ in the particles in Figure 5(c).

\section{Conclusions}

In order to achieve maximum sintered density in the fabrication of Al-rich Al-Fe (Al-6.23 wt.\% Fe) metal matrix composites, optimum sintering temperature is found to be $550^{\circ} \mathrm{C}$ (which is $75 \%$ of the melting temperature of the matrix). Sintered density increases with increase in sintering temperature up to $550^{\circ} \mathrm{C}$ and decreases with further increase in temperature. A drop in sintered density, that is, swelling, is found at $590^{\circ} \mathrm{C}$ for all the green compacts. This swelling behaviour is considered to occur due to the formation of excess vacancies and Al-Fe solid solution of lower density. These phenomena can be well described with the help of the Kirkendall effect. Sintered density is found to increase with a higher rate for the green compacts of lower green density as compared to that of higher green density. Maximum green density of the compacts, that is, $\sim 2.5 \mathrm{~g} / \mathrm{cm}^{3}$, is found at $399 \mathrm{MPa}$ which is $\sim 82 \%$ of the theoretical density. As expected, the green density of the compacts increases with increase in compaction pressure whereas the rate of increase in green density decreases with the increasing compaction pressure.

\section{Acknowledgments}

The authors are thankful to Dr. Rakesh Bhatnagar (Faculty, UIET, CSJM University, Kanpur) and DMSRDE DRDO, Kanpur, India, for the technical discussion and support throughout the work.

\section{References}

[1] M. K. Surappa, "Aluminium matrix composites challenges and opportunities," Sadhana, vol. 28, p. 319, 2003.
[2] S. Mohan and S. Srivastava, "Surface behaviour of asCast AlFe intermetallic composites," Tribology Letters, vol. 22, no. 1, pp. 45-51, 2006.

[3] A. Heinz, A. Haszler, C. Keidel, S. Moldenhauer, R. Benedictus, and W. S. Miller, "Recent development in aluminium alloys for aerospace applications," Materials Science and Engineering, vol. 280, no. 1, pp. 102-107, 2000.

[4] J. M. Torralbaa, C. E. da Costab, and F. Velasco, "P/M aluminum matrix composites: an overview," Journal of Materials Processing Technology, vol. 133, pp. 203-206, 2003.

[5] J. R. Pickens, "Aluminium powder metallurgy technology for high-strength applications," Journal of Materials Science, vol. 16, no. 6, pp. 1437-1457, 1981.

[6] Y. B. Liu, S. C. Lim, L. Lu, and M. O. Lai, "Recent development in the fabrication of metal matrix-particulate composites using powder metallurgy techniques," Journal of Materials Science, vol. 29, no. 8, pp. 1999-2007, 1994.

[7] S.-J. L. Kang, Sintering: Densification, Grain growth and Microstructure, Jorden Hill, Oxford, UK, 1st edition, 2005.

[8] D. Bouvard, "Densification behaviour of mixtures of hard and soft powders under pressure," Powder Technology, vol. 111, no. 3, pp. 231-239, 2000.

[9] M. Braginsky, V. Tikare, and E. Olevsky, "Numerical simulation of solid state sintering," International Journal of Solids and Structures, vol. 42, no. 2, pp. 621-636, 2005.

[10] J. Svoboda and H. Riedel, "New solutions describing the formation of interparticle necks in solid-state sintering," Acta Metallurgica et Materialia, vol. 43, no. 1, pp. 1-10, 1995.

[11] C. L. Martin and D. Bouvard, "Study of the cold compaction of composite powders by the discrete element method," Acta Materialia, vol. 51, no. 2, pp. 373-386, 2003.

[12] T. Kraft and H. Riedel, "Numerical simulation of solid state sintering; model and application," Journal of the European Ceramic Society, vol. 24, no. 2, pp. 345-361, 2004.

[13] G. S. Upadhyaya, "Future directions in sintering research," Science of Sintering, vol. 43, no. 1, pp. 3-8, 2011.

[14] G. S. Upadhyaya, "Some issues in sintering science and technology," Materials Chemistry and Physics, vol. 67, no. 13, pp. 1-5, 2001.

[15] Y.-S. Kwon and A. Savitskii, "Solid-state sintering of metal powder mixtures," Journal of Materials Synthesis and Processing, vol. 9, pp. 299-317, 2001.

[16] H.-Z. Kang and C. -T. Hu, "Swelling behavior in reactive sintering of Fe-Al mixtures," Materials Chemistry and Physics, vol. 88, no. 2-3, pp. 264-272, 2004.

[17] N. Showaiter and M. Youseffi, "Compaction, sintering and mechanical properties of elemental $6061 \mathrm{Al}$ powder with and without sintering aids," Materials \& Design, vol. 29, no. 4, pp. 752-762, 2008.

[18] M. N. Rahaman, Ceramic Processing and Sintering, CRC Press, New York, NY, USA, 2nd edition, 2005.

[19] H. Masuda, K. Higashitani, and H. Yoshida, Powder Technology Handbook, Taylor and Francis group, New york, NY, USA, 3rd edition, 2006.

[20] H. Djohari, J. I. Martínez-Herrera, and J. J. Derby, “Transport mechanisms and densification during sintering: I. Viscous flow versus vacancy diffusion," Chemical Engineering Science, vol. 64, no. 17, pp. 3799-3809, 2009.

[21] H. Djohari and J. J. Derby, "Transport mechanisms and densification during sintering: II. Grain boundaries," Chemical Engineering Science, vol. 64, no. 17, pp. 3810-3816, 2009.

[22] P. F. Stableinjr and G. C. Kuczynskit, "Sintering in multicomponent metallic systems," Acta Metallurgica, vol. 11, no. 12, pp. 1327-1337, 1963. 
[23] R. E. Reed-Hill and R. Abbaschian, Physical Metallurgy Principles, PWS Pub. Co., Boston, UK, 3rd edition, 1994.

[24] R. W. Balluffi and L. L. Seigle, "Growth of voids in metals during diffusion and creep," Acta Metallurgica, vol. 5, no. 8, pp. 449-454, 1957.

[25] R. S. Barnes, "The climb of edge dislocations in face-centred cubic crystals," Acta Metallurgica, vol. 2, no. 3, pp. 380-385, 1954.

[26] K. I. Hirano, R. P. Agarwala, and M. Cohen, "Diffusion of iron, nickel and cobalt in aluminum," Acta Metallurgica, vol. 10, no. 9, pp. 857-863, 1962.

[27] A. Olszówka-Myalska, J. Szala, and J. Cwajna, "Characterization of iron aluminides formed in situ in an aluminium matrix composite," Materials Characterization, vol. 56, no. 4-5, pp. 379-383, 2006.

[28] D. K. Mukhopadhyay, C. Suryanarayana, and F. H. Froes, "Structural evolution in mechanically alloyed Al-Fe powders," Metallurgical and Materials Transactions A, vol. 26, no. 8, pp. 1939-1946, 1995. 

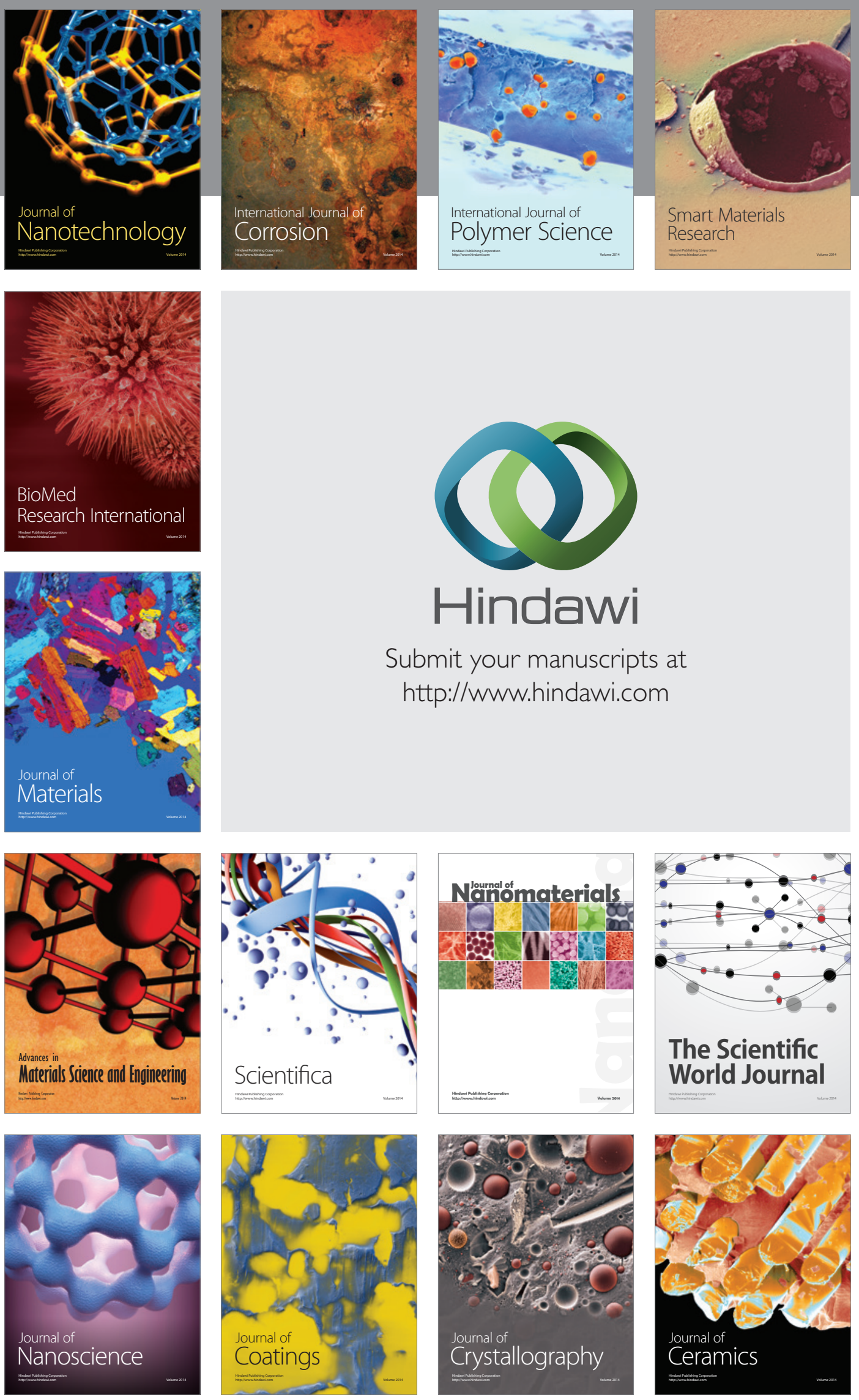

The Scientific World Journal

Submit your manuscripts at

http://www.hindawi.com

\section{World Journal}

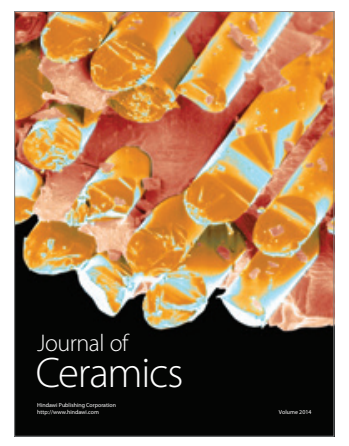

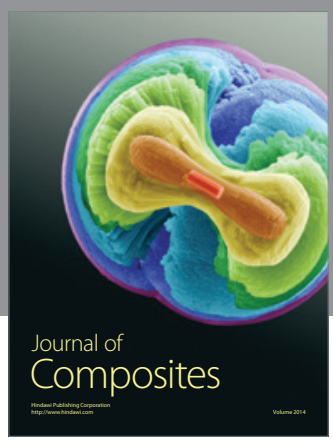
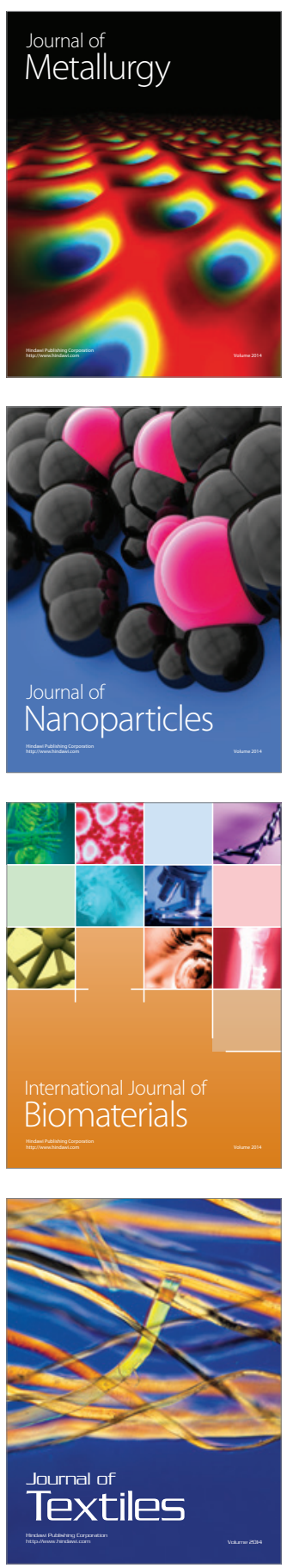